\title{
How important are shock waves to single-bubble sonoluminescence?
}

\author{
H. Y. Cheng, ${ }^{1}$ M.-C. Chu, ${ }^{1}$ P. T. Leung, ${ }^{1}$ and L. Yuan ${ }^{1,2}$ \\ ${ }^{1}$ Department of Physics, The Chinese University of Hong Kong, Shatin, N.T., Hong Kong \\ ${ }^{2}$ LNM, Institute of Mechanics, Academia Sinica, Beijing 100080, People's Republic of China
}

(Received 19 November 1997; revised manuscript received 26 May 1998)

\begin{abstract}
By solving numerically the full set of hydrodynamic equations governing the pulsation of a bubble, we show that shock waves are often absent in a stable sonoluminescing bubble. Nevertheless, for a wide range of physical parameters, a continuous compressional wave emerges and heats up the bubble, and the resulting black-body radiations have pulse heights and widths that agree with experimental data. Shock waves, being much less robust, are not essential for stable single-bubble sonoluminescence. [S1063-651X(98)50908-7]
\end{abstract}

PACS number(s): 78.60.Mq, 47.40.-x, 44.10.+i, 43.25.+y

The remarkable discovery that acoustic energy can be converted to light through an oscillating air bubble trapped in water has ignited widespread interest in single-bubble sonoluminescence (SBSL). Under certain conditions, a narrow and regular flash of light with a width of about 50-250 ps is emitted in each cycle of the bubble oscillation [1,2]. Such a conversion of sound into light represents a high concentration of energy, and it has been speculated that the temperature and pressure at the center of the bubble could be high enough to ignite thermonuclear fusion [3].

Previous calculations based on inviscid spherical hydrodynamics [3-7] suggested that a converging shock produced high temperature and pressure and the reflected diverging shock quenched them in picosecond time scale $[3,4,8]$. This model could explain the emergence of a picosecond time scale as well as the large energy concentration, but there are still many unanswered questions, such as whether the shock waves are stable and robust. The effects of transport processes, surface tension, the equation of state (EOS), and the compressibility of liquid were also largely unknown and ignored.

An important clue to the mechanism of SBSL is its sensitivity to the gas content: apparently the presence of noble gas is essential. This can be explained by the argonrectification theory [9], in which air molecules dissociate and react and leave the bubble in the pre-SBSL stage, and argon becomes the dominant bubble content when stable lightemission takes place. If shock-heating is essential to SBSL, then one expects that shock waves should develop easily in an argon bubble. A major goal in this Rapid Communication is to study and compare the relevance of shock waves in both air and argon SBSL.

To determine whether shock waves are important to SBSL, and to study the sensitivity of the process to the experimental conditions, a reliable hydrodynamic simulation that takes into account various physical processes affecting the bubble, including diffusive transport and different bubble content, surface tension, and liquid compressibility, is essential. In this Rapid Communication, based on a comprehensive numerical study of the bubble hydrodynamics, we show that shock waves are absent in a large parameter range of argon SBSL, in agreement with an earlier calculation by Vuong and Szeri [10]. Rather, a smooth compressional wave emerges naturally in a collapsing bubble, and a large fraction of the bubble content is heated to moderate temperatures for a short duration. This heating mechanism is robust and effective even without the formation of shock waves. Assuming that the bubble content radiates like a black body and is optically thin, we obtain a radiation power comparable to the experimental result. For an air bubble, whether there are shock waves depends sensitively on many physical parameters and the EOS used. Our results suggest that the existence of shock waves is not needed for stable SBSL, though they may play a role in argon-rectification.

Assuming local equilibrium and ignoring mass diffusion, the dynamics of a spherical bubble is described by the compressible Navier-Stokes equations:

$$
\frac{\partial \mathbf{Q}}{\partial t}+\frac{\partial \mathbf{F}(\mathbf{Q})}{\partial x}=\mathbf{S}(\mathbf{Q})+\mathbf{S}_{v}(\mathbf{Q})
$$

where $x \equiv r / R(t)$ has been used to transform the equations to a fixed domain $x \in[0,1]$. Here $\mathbf{Q}=R(\rho, \rho v, E)^{T}$, with $E$ $=\rho\left(e+\frac{1}{2} v^{2}\right)$ the total energy per unit volume, $R, \rho, v, P, T$, and $e$ the bubble radius, density, radial velocity, pressure, temperature, and internal energy per unit mass respectively. The inviscid fluxes are $\mathbf{F}=\left[\rho v-\rho x \dot{R}, \rho v^{2}+P-\rho v x \dot{R}\right.$, $(E+P) v-E x \dot{R}]^{T}$, and the spherical sources are $\mathbf{S}$ $=-2 v[\rho, \rho v,(E+P)]^{T} / x$. The diffusive transport terms are given by $\mathbf{S}_{v}=\left\{0, \partial_{x}\left(x^{2} \tau_{r r}\right)+x \tau_{r r}, \partial_{x}\left[x^{2}\left(v \tau_{r r}+k \partial_{x} T /\right.\right.\right.$ $R)]\}^{T} / x^{2}$, where $\tau_{r r}=4 \mu\left(\partial_{x} v-v / x\right) / 3 R$ is the normal viscous stress, $k$ is the coefficient of thermal conductivity, and $\mu$ is the dynamic viscosity.

The hydrodynamics of the bubble is affected by the compressibility of the gas, as characterized by the EOS. Physically speaking, a smaller compressibility makes it harder for shock waves to develop $[10,11]$. We have used a range of EOS's in our study. The hard-core van der Waals EOS (VEOS) approximates both air and argon properties better than an ideal gas EOS at high pressure/density, such as those expected inside a collapsing bubble [12]. We also used two other more complicated EOS's: one for nitrogen (NEOS), which represents air [3,7], and one for argon (AEOS) [13]. They both include effects of vibrational excitation, dissociation and ionization of molecules, as well as intermolecular potentials, but without a hard core. Whether a hard core is 
included affects directly the compressibility, and we expect the hydrodynamics to behave accordingly.

The Navier-Stokes equations are coupled to the RayleighPlesset (RP) equation that describes the bubble wall motion. Several forms of the RP equation have been used in the literature, which differ in the terms that account for the compressibility of the liquid surrounding the bubble, characterized by the bubble-wall Mach number $M \equiv \dot{R} / c_{l}$. A formulation (RP3) that includes terms to first order in $M$ and allows for a variable speed of sound in the liquid $c_{l}$ [14] gives

$$
\frac{1-M}{1+M} R \ddot{R}+\frac{3-M}{2(1+M)} \dot{R}^{2}=H_{b}-\frac{P_{s}\left(t^{\prime}\right)}{\rho_{\infty}}+\frac{t_{R} \dot{H}_{b}}{1+M} .
$$

Here overdots denote time derivatives, $t_{R} \equiv R / c_{l}, t^{\prime} \equiv t$ $+t_{R}, P_{s}(t)=-P_{a} \sin (\omega t)$ the pressure of the sound field with frequency $\omega$ and amplitude $P_{a}$, and $H_{b}$ is the enthalpy of the liquid. The pressure $P_{g}(R, t)$ on the gas side of the bubble wall exceeds the pressure $P_{b}(t)$ on the liquid side of the bubble wall by the effects of surface tension and the normal component of viscous stresses:

$$
P_{g}(R, t)-\left.\tau_{r r}\right|_{r=R}=P_{b}(t)+\frac{4 \eta \dot{R}}{R}+\frac{2 \sigma}{R},
$$

where $\sigma$ is the surface tension and $\eta$ the dynamic viscosity of the liquid. For water, $c_{l}$ and $H_{b}$ are obtained using an EOS of the modified Tait form $[15,16]$. Two other commonly used forms of the RP equation are simplified versions of RP3. Taking a constant speed of sound $c_{l}$ and liquid density, one obtains the Keller equation (RP2) [17]. An even simpler equation (RP1) results when $M=0$ is assumed [4,5,10,12].

The energy equation for the liquid is [10]

$$
\frac{\partial T_{l}}{\partial t}+v_{l} \frac{\partial T_{l}}{\partial r}=D_{l} \frac{1}{r^{2}} \frac{\partial}{\partial r}\left(r^{2} \frac{\partial T_{l}}{\partial r}\right)
$$

where $T_{l}, v_{l}$, and $D_{l}$ are the temperature, velocity, and thermal diffusion coefficient of the liquid.

We apply a second-order TVD scheme [18] to the convective terms of Eq. (1), and a second-order predictorcorrector method for the time discretization of both the gas dynamics and the RP equation. The implicit Crank-Nicolson method is used to solve Eq. (4). The variable time step is adjusted according to the Courant number. Our results are based on calculations with 400 points inside the bubble and 50 points in the liquid. Details of the numerical scheme as well as a systematic study of the sensitivities of the hydrodynamic solutions to various physical parameters are reported in a separate paper [11]. We calculated the bubble oscillation for one period of the acoustic forcing starting from an equilibrium radius $R_{0}$. We assume that the heat conductivity is linear in $T$ [19] and adopt the gas viscosity used in Ref. [10]. Here, we report results for $R_{0}=4.5 \mu \mathrm{m}$, $T_{0}=300 \mathrm{~K}, \omega / 2 \pi=26.4 \mathrm{kHz}$, and standard values for other parameters [11]. Whereas $R_{0}$ depends on the driving pressure, our conclusions remain unchanged for a range of $R_{0}$, corresponding to those observed experimentally.

We first computed for air with three driving pressures, $P_{a}=1.275,1.35$, and $1.4 \mathrm{~atm}$, which approximately span the
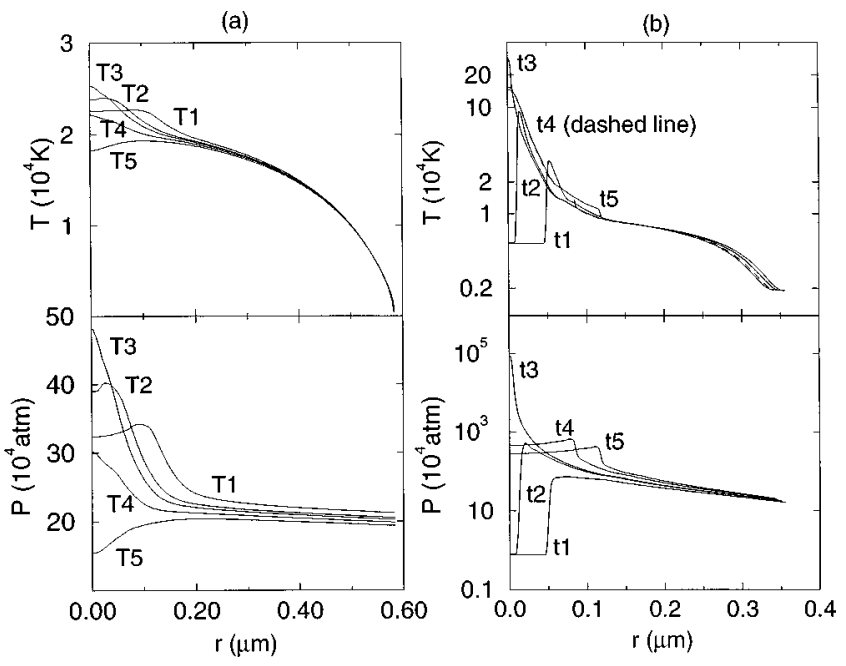

FIG. 1. Snapshots of the spatial profiles of pressure $P$ and temperature $T$ for an air bubble driven with a sound amplitude of $P_{a}$ $=1.35 \mathrm{~atm}$. (a) Results with VEOS are shown at $T 1=18 \mathrm{ps}, T 2$ $=20.1 \mathrm{ps}, T 3=21 \mathrm{ps}, T 4=22.1 \mathrm{ps}$, and $T 5=24 \mathrm{ps}$, taking the time at minimum bubble radius as zero. (b) Same as (a), but with NEOS and $t 1=-26.6 \mathrm{ps}, \quad t 2=-23.9 \mathrm{ps}, \quad t 3=-23.5 \mathrm{ps}, \quad t 4$ $=-20.4 \mathrm{ps}$, and $t 5=-18.4 \mathrm{ps}$. The shock front moves inward towards $r=0$ at $t 1$ and $t 2$ and outward at $t 4$ and $t 5$.

range of stable SL observed experimentally [20]. In Fig. 1(a), we show snapshots of the spatial profiles of several thermodynamical variables around the instance of maximum temperature and minimum radius inside an air bubble (VEOS) driven at $P_{a}=1.35 \mathrm{~atm}$. A compressional wave is formed, which produces high temperature, pressure, and density, even though no shock waves are present. For comparison, a solution with shock is shown in Fig. 1(b) obtained with NEOS. Notice that both the temperature and pressure jump by about two orders of magnitude within a narrow shock front, whereas they vary smoothly across a compressional wave. The difference in the results arises from the fact that NEOS has a larger compressibility, and the bubble collapses to a smaller volume and higher pressure. As an orderof-magnitude estimate for the light pulse, we calculated the total radiation power for both EOS's, assuming a black body model with an optically thin medium [3,21]. The pulse widths are 50 and 5 ps for VEOS and NEOS, respectively [Fig. 2(a)]. Whereas the former is comparable to the experimental values of between 50 to 250 ps [2], the latter one seems too narrow due to the focusing of a shock wave. The pulse heights are about 2.5 and $5 \mathrm{~mW}$ respectively, in fair agreement with experimental data.

Putting aside the stability problem for converging shock waves [22], whether a shock wave can develop depends sensitively not only on the EOS as we discussed, but also on the driving pressure, surface tension, the compressibility of the liquid, and heat conductivity. For example, the shock-free results with VEOS for $P_{a}=1.35$ atm are obtained with a surface tension of $\sigma=0.0725 \mathrm{~kg} \mathrm{~s}^{-2}$, the value for pure water. If we lower the surface tension to $0.05 \mathrm{~kg} \mathrm{~s}^{-2}$, as typically used in previous calculations to account for impurities [1], a shock wave develops. The surface tension limits the maximum radius in the expansion phase and hence reduces the violence of the bubble-wall collapse. We can study the effects of the liquid compressibility on shock formation by 


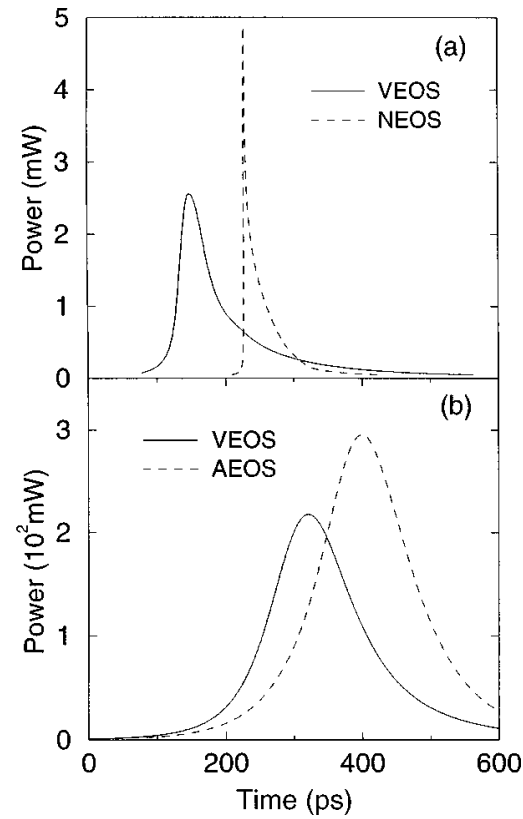

FIG. 2. Black-body radiation power vs time. (a) For an air bubble with NEOS (dashed line) and VEOS (solid line), starting from 250 (150) ps before the instance of minimum bubble radius. Results are from the same calculations as in Fig. 1. (b) Same as (a), but for an argon bubble. The dashed (solid) line is for AEOS (VEOS), starting from 407 (327) ps before the instance of minimum bubble radius.

comparing the results using different versions of the RP equation. We find that if the liquid compressibility is ignored, as in RP1, shock waves develop easily. However, when some compressibility corrections are included, as in RP3, the bubble wall compresses the gas less violently, and shock waves are weakened to compressional waves. Viscosity and thermal conductivity also tend to smear out discontinuities $[6,10]$. Our results indicate that with realistic parameters for SBSL, viscosity, heat conductivity, surface tension, and the liquid compressibility together strongly suppress shock formation.

Even though shock waves are often weakened to form smooth compressional waves, the radiation power is little affected. In particular, the compressional waves are already adequate to produce a sharp power peak with a width comparable to experimental data. The presence of shock waves only reduces the pulse width further, but it does not appear to be a necessary condition for stable SBSL. In fact, the shockinduced pulse width seems to be too short compared with experimental data.

For an argon bubble, smooth compressional waves, rather than shock waves, are formed for all driving pressures between 1.275 and $1.4 \mathrm{~atm}$. We show in Fig. 2(b) the total radiation power and in Fig. 3 the spatial profiles of temperature and pressure, for an argon bubble driven at $P_{a}=1.35$ atm, using AEOS [13] and VEOS. There is no qualitative difference between the results using the two EOS's; there are no shock waves in either case. The larger ratio of specific heats of noble gases, and hence a smaller compressibility, makes it harder for shock waves to develop [10,11]. The AEOS does not include a hard core, and thus its compressibility is slightly larger than that of VEOS. This allows for
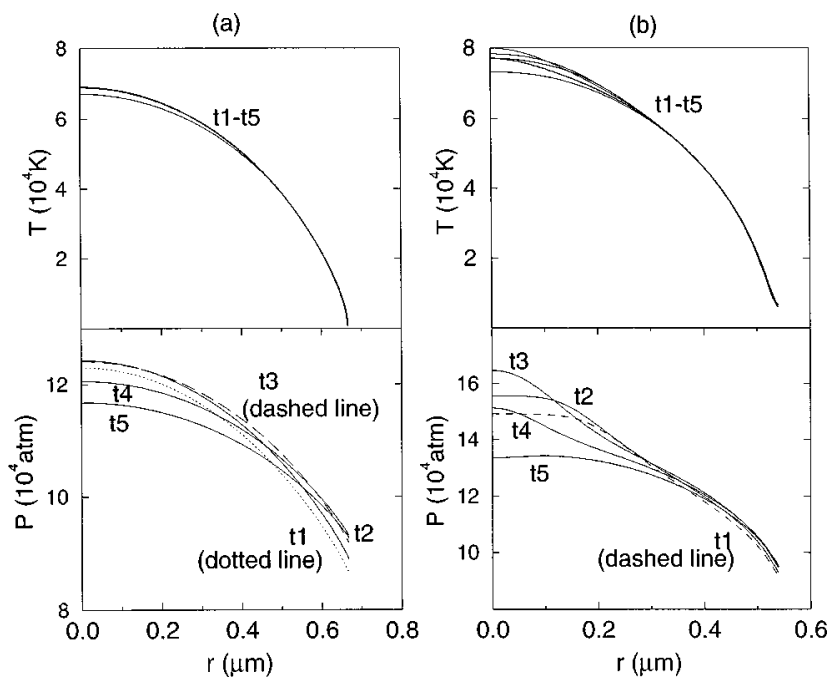

FIG. 3. Same as Fig. 1, but for an argon bubble. Results for (a) VEOS and (b) AEOS are shown at $t 1=-18 \mathrm{ps}, t 2=-11 \mathrm{ps}, t 3$ $=0, t 4=11 \mathrm{ps}$, and $t 5=18.5 \mathrm{ps}$.

slightly steeper compressional waves in the results using AEOS compared to those in VEOS. The peak temperature can be about $80000 \mathrm{~K}$ without the formation of shocks. Both the width and magnitude of the radiated power are comparable to experimental data and generally larger than those of an air bubble. Table I summarizes the dependence of shock formation, peak radiated power, and the pulse width on the driving pressure and EOS.

The results for argon gas clearly show the importance of heating due to compressional waves [10]. The hydrodynamic process is neither violent enough as to launch an imploding shock nor trivially uniform, but rather in between. The compressional wave alone has already greatly enhanced the energy focusing in the bubble, and a relatively wide region of gas (compared to shock-heating) is heated up to moderately high temperatures.

The fact that shock waves are formed relatively easily in air or nitrogen, but not in argon, suggests that shock waves may occur during the transient argon-rectifying stage [23] of air bubble SBSL, when the bubble is being cleansed of air

TABLE I. Shock waves and power pulses characteristics.

\begin{tabular}{ccclcc}
\hline \hline $\begin{array}{c}P_{a} \\
(\mathrm{~atm})\end{array}$ & Gas & EOS & $\begin{array}{c}\text { Shock } \\
\text { waves }\end{array}$ & $\begin{array}{c}\text { Peak power } \\
(\mathrm{mW})\end{array}$ & $\begin{array}{c}\text { FWHM } \\
\text { of power (ps) }\end{array}$ \\
\hline 1.275 & air & VEOS & No & 0.5 & 100 \\
& & NEOS & Yes & 0.6 & 3 \\
& argon & VEOS & No & 27.5 & 218 \\
& & AEOS & No & 28.5 & 231 \\
\hline 1.35 & air & VEOS & No & 2.5 & 50 \\
& & NEOS & Yes & 5 & 5 \\
& \multirow{2}{*}{ argon } & VEOS & No & 258 & 140 \\
& & AEOS & No & 298 & 148 \\
\hline 1.4 & air & VEOS & Yes & 5.5 & 15 \\
& & NEOS & Yes & 13.0 & 5 \\
& argon & VEOS & No & 776 & 97 \\
& & AEOS & No & 956 & 107 \\
\hline \hline
\end{tabular}


and hence the bubble content and the light intensity are changed. After the "turn on" time, the bubble becomes mostly argon, and shock waves are hardly formed. Nevertheless, smooth compressional waves develop, which continue to heat up the gas and induce light emission. This scenario, together with our findings that the radiation power of an argon bubble is higher than that in an air bubble under the same driving pressure, give a natural explanation to Löfstedt et al.'s observation that an air bubble undergoes a phase of low intensity and irregular light emission before entering a steady state emitting at higher intensity [23].

In conclusion, we have shown that shock waves are absent in the stable argon SBSL regime, and their formation in air bubble is very sensitive to various physical parameters and EOS's used. For both air and argon bubbles, smooth compressional waves are much more robust and are already adequate to explain the radiation power and pulse width of SBSL. Because they are so robust, we speculate that compressional waves are present even in asymmetric collapses [24], where the stability of converging spherical shock waves is particularly doubtful.

We are thankful for the support of the Hong Kong Research Grants Council under Grant No. CUHK 312/96P and a Chinese University Direct Grant (Project No. 2060093). L. Yuan is thankful for the support of Chinese University.
[1] D. F. Gaitan et al., J. Acoust. Soc. Am. 91, 3166 (1992); B. P. Barber et al., Phys. Rep. 281, 65 (1997), and references therein.

[2] B. Gompf et al., Phys. Rev. Lett. 79, 1405 (1997).

[3] W. Moss et al., Phys. Fluids 6 (9), 2979 (1994).

[4] C. C. Wu and P. H. Roberts, Phys. Rev. Lett. 70, 3424 (1993).

[5] L. Kondić, J. I. Gersten, and C. Yuan, Phys. Rev. E 52, 4976 (1995).

[6] M.-C. Chu and D. Leung, J. Phys.: Condens. Matter 9, 3387 (1997).

[7] W. Moss et al., Science 276, 1398 (1997).

[8] P. Jarman, J. Acoust. Soc. Am. 32, 1459 (1960).

[9] D. Lohse et al., Phys. Rev. Lett. 78, 1359 (1997).

[10] V. Q. Vuong and A. J. Szeri, Phys. Fluids 8, 2354 (1996).

[11] L. Yuan, H. Y. Cheng, M.-C. Chu, and P. T. Leung, Phys. Rev. E 57, 4265 (1998).

[12] R. Löfstedt, B. P. Barber, and S. J. Putterman, Phys. Fluids A 5, 2911 (1993).

[13] D. Young (private communication). The same EOS was also used in Ref. [7].
[14] V. Kamath and A. Prosperetti, J. Acoust. Soc. Am. 85, 1538 (1987).

[15] A. Prosperetti and A. Lezzi, J. Fluid Mech. 168, 457 (1996).

[16] R. H. Cole, Underwater Explosion (Princeton University Press, Princeton, 1948).

[17] J. B. Keller and M. Miksis, J. Acoust. Soc. Am. 68, 628 (1980).

[18] H. C. Yee, NASA Report No. TM 101088, 1989 (unpublished).

[19] V. Kamath and A. Prosperetti, J. Acoust. Soc. Am. 85, 1538 (1989).

[20] R. G. Holt and D. F. Gaitan, Phys. Rev. Lett. 77, 3791 (1996).

[21] Ya. B. Zeldovich and Yu. P. Raizer, Physics of Shock Waves and High-Temperature Hydrodynamic Phenomena (Academic Press, New York, 1966).

[22] A. K. Evans, Phys. Rev. E 54, 5004 (1996).

[23] R. Löfstedt et al., Phys. Rev. E 51, 4400 (1995).

[24] A. Prosperetti, J. Acoust. Soc. Am. 101, 2003 (1997); T. Lepoint et al., ibid. 101, 2012 (1997). 